Article

\title{
The Mayan Tropical Rainforest: An Uncharted Reservoir of Tritrophic Host-Fruit Fly-Parasitoid Interactions
}

\author{
Maurilio López-Ortega ${ }^{1 * *(\mathbb{D})}$, Francisco Díaz-Fleischer ${ }^{1}{ }^{\mathbb{C}}$, Jaime C. Piñero ${ }^{2} \mathbb{C}$, \\ José René Valdez-Lazalde ${ }^{3}$, Manuel Hernández-Ortiz ${ }^{1}$ and Vicente Hernández-Ortiz ${ }^{4}$ (i) \\ 1 Instituto de Biotecnología y Ecología Aplicada (INBIOTECA), Universidad Veracruzana, Xalapa, \\ 91090 Veracruz, Mexico; fradiaz@uv.mx (F.D.-F.); manuelhdezo94@gmail.com (M.H.-O.) \\ 2 Stockbridge School of Agriculture, University of Massachusetts, Amherst, MA 01003, USA; \\ jpinero@umass.edu \\ 3 Colegio de Postgraduados, Postgrado en Ciencias Forestales, Montecillo, 56230 Texcoco, Mexico; \\ valdez@colpos.mx \\ 4 Red de Interacciones Multitróficas, Instituto de Ecología A.C. Xalapa, 91073 Veracruz, Mexico; \\ vicente.hernandez@inecol.mx \\ * Correspondence: maulopez@uv.mx
}

Received: 26 June 2020; Accepted: 30 July 2020; Published: 3 August 2020

Simple Summary: Tropical rainforest can provide various ecological services to adjacent agricultural environments, including maintaining and amplifying the numbers of beneficial insects. However, forest fragmentation and the selective cutting of indigenous trees used by native species of fruit flies and their parasitoids, degrades the potential of forests to provide ecological services to agriculture. Over a two-year period, we surveyed natural areas of the Mayan rainforest in Quintana Roo, Mexico. We found 11 species of native fruit flies belonging to the genus Anastrepha associated with 25 species of fruits belonging to ten plant families. We report the first records of 10 host plant species of the genus Anastrepha. We also report a new undescribed species of Anastrepha. The interaction between fruit flies and their parasitoids with host plants depends on fruit availability, which is crucial for the survival of each of these species. Our findings indicate that the areas of the Mayan rainforest surveyed represent a highly important reservoir for the diversity of native parasitoids spatially and temporally that are practically absent in fruits of cultivated plants. Conserving the landscape of the Mayan rainforest is important not only for species conservation, but also for the maintenance of fruit fly host plants of biological control agents in orchard agroecosystems in southeastern Mexico.

Abstract: Over a two-year period, we surveyed natural areas of the Mayan rainforest in Quintana Roo, Mexico. We found 11 species of Anastrepha Schiner (Diptera: Tephritidae) infesting 25 species of fruits belonging to ten plant families. We report the first records of 10 host plant species of the genus Anastrepha, which include the first report of a plant family (Putranjivaceae) serving as host of Anastrepha fraterculus (Wiedemann) infesting Drypetes lateriflora (Sw.) Krug and Urb. (Putranjivaceae). Pouteria reticulata (Engl.) Eyma (Sapotaceae) was found, for the first time, to be infested by Anastrepha serpentina (Wiedemann) and by a new undescribed species of Anastrepha. We also report Casimiroa microcarpa Lundell (Rutaceae) as a possible ancestral host for the Mexican fruit fly, Anastrepha ludens (Loew), in Central America. The family Sapotaceae was the best-represented host group with three fruit fly species recovered: A. serpentina, an economically-important species, found in eight host plants, and $A$. hamata and $A$. sp. (new species). We recorded six species of koinobiont parasitoids: Doryctobracon areolatus Szepligeti, Utetes (Bracanastrepha) anastrephae Viereck, Opius hirtus Fisher, and Doryctobracon zeteki Musebeck, (all Braconidae), and Aganaspis pelleranoi (Brethés) and Odontosema anastrephae Borgmeier, (both Figitidae). All these parasitoid species represent at least a new report for their host plants. Of the whole parasitoid community, D. areolatus was the most 
important parasitoid species with $52.7 \%$ of presence in 12 host plant species, parasitizing six fruit fly species. The interaction between fruit flies and their parasitoids with host plants depends on fruit availability, which is crucial for the survival of each of these species. Conserving the landscape of the Mayan rainforest is important not only for species conservation, but also for the maintenance of fruit fly host plants in orchard agroecosystems in southeastern Mexico.

Keywords: host-plants; Anastrepha; tropics; conservation; frugivory; biodiversity

\section{Introduction}

Herbivorous insects have a powerful influence on plant abundance and distribution, as well as on the composition of plant communities [1,2]. The study of interactions between insects and fruits is one of the main challenges for understanding the reproductive success of many angiosperms because the damage caused by insects can cause the abortion of a wide variety of fruits [3,4]. Fruit-eating insects can influence the production of seeds, due to direct damage, and by indirect damage through biochemical changes that cause premature ripening of the fruit or increased protein levels. For example, wild tobacco, Solanum mauritianum Scop (Solanaceae), fruits infested by Dacus cacuminatus (Hering) (Diptera: Tephritidae) are reported to contain twice the levels of proteins and essential amino acids when compared to uninfested fruits [5]. Despite the ongoing loss of the original habitat in tropical ecosystems and the resulting detrimental effects on biodiversity [6,7], these ecosystems still harbor substantial numbers of potential host plants in practically every biological form, including herbs, vines, shrubs, and trees.

The great diversity of plants that occurs in tropical rainforests suggests the existence of a high diversity of tephritid fruit flies. The Neotropical genus Anastrepha exhibits great richness, estimated in 283 known species, some of which are pests of economic significance [8]. In Mexico and various countries of Central and South America, numerous samples of wild and cultivated tropical fruits have been examined in order to determine their seasonal phenology and infestation levels, produced mainly by Anastrepha species. In numerous occasions, such insect-plant biological interactions were recorded for the first time [9-17]. There are few systematic studies on trophic interactions between frugivorous tephritids and their host plants in natural environments in the Americas [18-21], unlike those carried out in agroecosystems where only a few fruit fly species are found associated with cultivated fruits [22,23]. There is an implicit depletion of these natural systems, in terms of both taxonomic richness and host-fruit fly interactions, due to the introduction of non-native fruit trees to the Americas, where they have recently experienced a trophic adaptation and new herbivore/parasitoid interactions.

In Mexico, the most recent reports include 39 described species of Anastrepha, which inhabit different regions of the country [24]. Some fly species, such as Anastrepha tehuacana Norrbom reproduce on plants that are endemic to xeric environments of central Mexico. Currently, A. tehuacana is considered a threatened species [16]. The identification of native parasitoids requires an intensive analysis of native and exotic fruits in order to verify the association between fly and parasitoid species and their host plants. A large guild of native parasitoids associated with Anastrepha spp. has been discovered in recent years [13,19,20,25,26]. Most parasitoid species identified, thus far belong to the family Braconidae, which are important in the suppression of natural populations of fruit flies and are of great interest in biological control techniques for fruit flies that cause severe economic losses in commercial orchards, due to the specificity to their hosts [27-29]. The parasitoid guild known in Mexico comprises 15 genera from six families represented by native species, as well as three exotic species, for which there are reports of proportions of parasitism and the range of hosts used for those fruit fly communities [19,30-35].

Recent studies have emphasized the importance of tropical rainforests in relation to trophic interactions among wild fruits, fruit flies, and their native parasitoids in those communities $[18,19,36,37]$. Studies aimed at increasing our knowledge of the diversity of Anastrepha fruit flies in natural habitats are fundamental for a better understanding of ancestral and more recent (i.e., in agroecosystems) trophic 
interactions. The main objective of this study was to identify the interactions between fruit fly species and their parasitoids in a fragment of the Mayan rainforest in the state of Quintana Roo, in the southeast region of México. We conducted intensive surveys of available fruits present throughout two annual cycles along two transects of the rainforest in order to identify the natural tri-trophic interactions represented by fruit fly species-plant-parasitoids, as well as to estimate their infestation rates and degree of parasitism.

\section{Materials and Methods}

\subsection{Study Site}

The study was conducted in natural areas of the Mayan rainforest in the municipalities of Felipe Carrillo Puerto and José María Morelos, in the state of Quintana Roo (Mexico). The predominant vegetation in these areas is characteristic of medium semi-evergreen forests sensu [38], where two tree layers can be distinguished: An upper layer, with characteristic elements, such as Pseudobombax ellipticum (Kunth) Dugand), Simarouba glauca DC, Cedrela odorata L., Swietenia macrophylla King, among others; and a middle layer, where we usually find Metopium brownei (Jacq.) Urb.), Manilkara zapota (L.) P. Royen, Sickingia salvadorensis (Standl.), Brosimun alicastrum Sw, Malmea depressa (Baill) R.E. Fries, and Gymmanthes lucida Swart [39].

The sampling area was established by using two transects of rural pathways and roads. The first transect (length: $154 \mathrm{~km})$ comprised the ejidos of Dzula $\left(19^{\circ} 35^{\prime} \mathrm{N}, 88^{\circ} 24^{\prime} \mathrm{W}, 28\right.$ masl) and $\mathrm{X}$ Pichil (19 $\left.46^{\prime} \mathrm{N}, 88^{\circ} 31^{\prime} \mathrm{W}, 47 \mathrm{masl}\right)$, in the municipality of Carrillo Puerto, and the ejidos of San Antonio Tuk $\left(19^{\circ} 45^{\prime} \mathrm{N}, 88^{\circ} 41^{\prime} \mathrm{W}, 11 \mathrm{masl}\right)$ and Xumuluc $\left(19^{\circ} 34^{\prime} \mathrm{N}, 88^{\circ} 31^{\prime} \mathrm{W}, 6\right.$ masl), in the municipality of José María Morelos ( $\left(9^{\circ} 44^{\prime} \mathrm{N}, 88^{\circ} 42^{\prime} \mathrm{W}, 54\right.$ masl) (Figure 1 Transect 1). The second transect (length: $230.5 \mathrm{~km})$ comprised the ejidos of Dzula, Laguna Kana $\left(19^{\circ} 21^{\prime} \mathrm{N}, 88^{\circ} 24^{\prime} \mathrm{W}, 40 \mathrm{masl}\right)$, Santa María (19 $21^{\prime} \mathrm{N}, 88^{\circ} 24^{\prime} \mathrm{W}, 26$ masl), and X-hazil $\left(19^{\circ} 20^{\prime} \mathrm{N}, 88^{\circ} 07^{\prime} \mathrm{W}, 27 \mathrm{masl}\right)$, in the municipality of Carrillo Puerto (Figure 1 Transect 2).

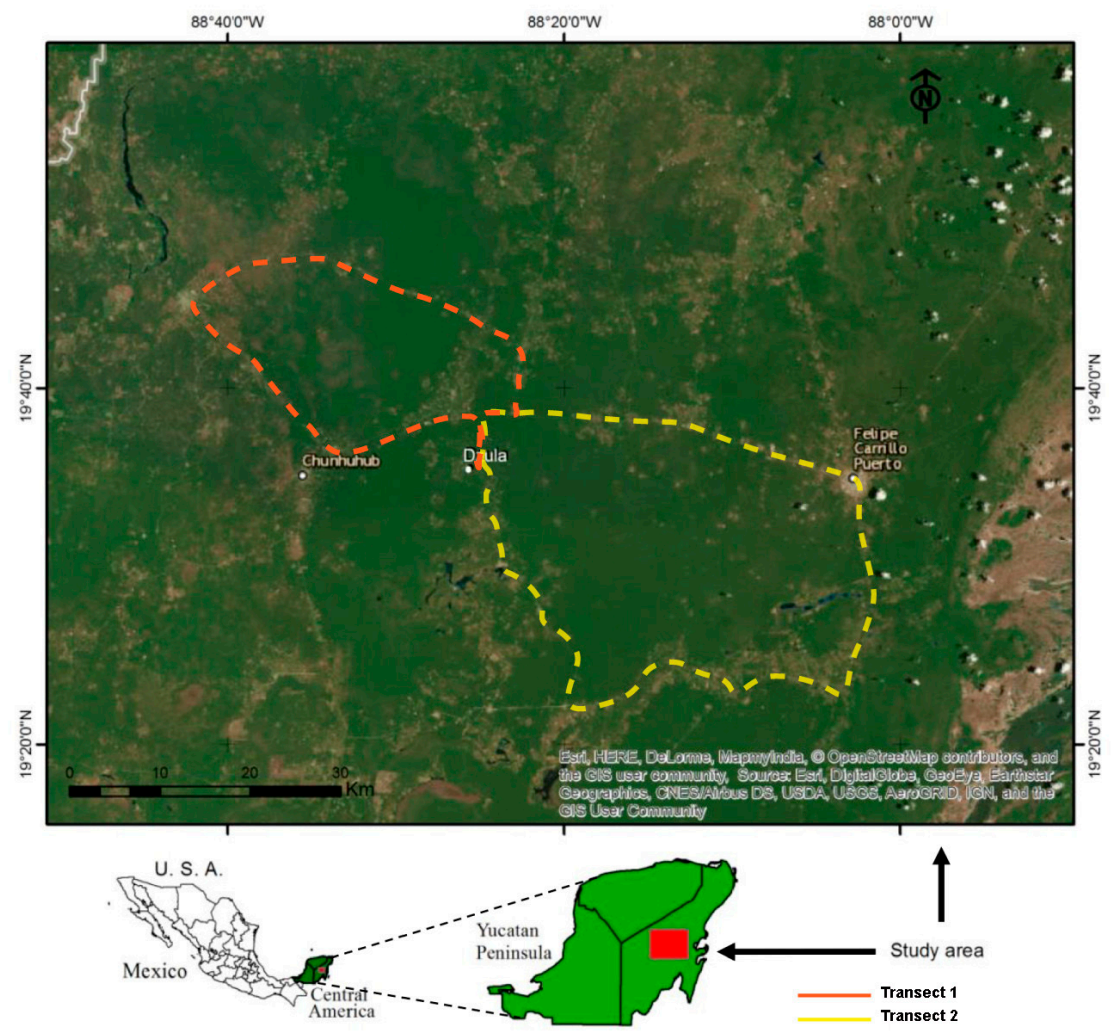

Figure 1. Map showing the location of the study area and an image showing the collection transects 
(Transect 1: red dashed line and Transect 2: yellow dashed line) in natural areas of the Mayan rainforest in Quintana Roo, Mexico.

\subsection{Collection and Processing of Fruit Samples}

During a biennial period, from March 2015 to December 2017, we carried out monthly samplings of available (ripe or unripe) fruits sampled from native and introduced plants along both transects. For each transect, there were about 10-12 stops, and for each stop, we spent about 90 min searching for available fruit. The fruits were either, cut directly from the plants (whenever possible using a tree pruner with a saw blade attached to a 4-m long wooden pole (Coronatoolsusa.com) or picked up when fallen, due to ripeness or damage by an insect. Fruits sampled were not decomposed or partially eaten by animals (Figure 2A). Each fruit sample was placed inside $50 \times 80 \mathrm{~cm}$ organdy cloth bag. We also obtained botanical samples for subsequent identification, as well as in situ photographs with a professional camera (Canon EOS 70D, Canon Inc., Tokyo, Japan).

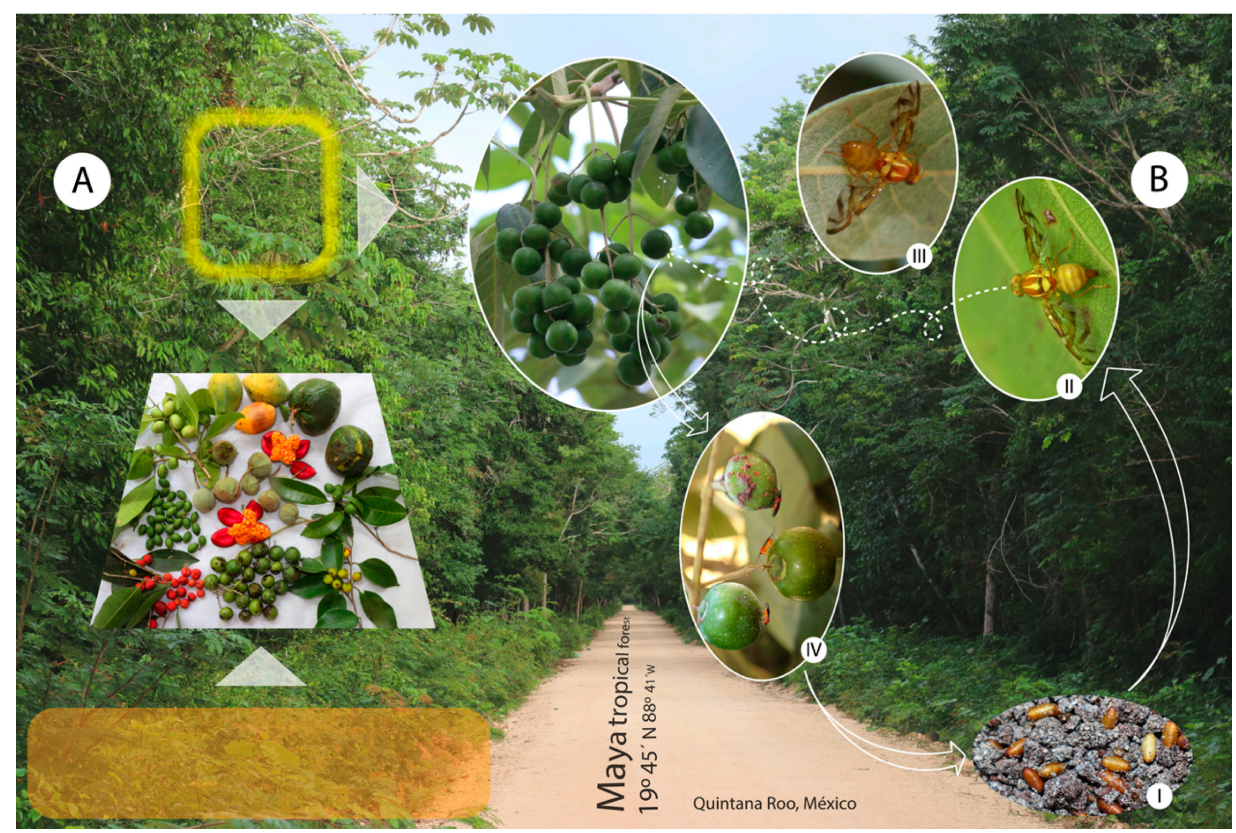

Figure 2. (A) Fruit collection methods: Arrows show where fruit was collected above and below the trees. (B) Insect life cycle in Vitex gaumeri fruits; fruits were found to be infested by Anastrepha ampliata. (BI) Dipteran larvae emerge from the fruits and fall to the ground in order to bury into the soil to pupate. (BII) A. ampliata female and (BIII) male, (BIV) Parasitoid species.

Each fruit sample was weighed in the nearest location, and placed in plastic containers with a metal grid, which rested on large plastic containers provided with sterilized river sand at the bottom as pupation substrate. These containers were placed on shelves inside an open-sided roofed shack, provided by local cooperators. This structure protected the fruit from rain and direct sunlight. The sand was regularly inspected, and all pupae recovered were placed in 500-mL labeled plastic cups covered with cloth. After one week, the fruit samples and the recovered pupae were transported to the Bioprospecting Laboratory of the Instituto de Biotecnología y Ecología Aplicada (INBIOTECA) (Xalapa, Veracruz, Mexico) for further processing. From each sample, we separated and weighed 40 fruits individually in order to obtain an accurate estimation of the infestation index. Depending on their size, fruits were placed in $100 \mathrm{~mL}, 250 \mathrm{~mL}$ or $500 \mathrm{~mL}$ plastic containers containing moist sand as pupation substrate. The containers were covered with a cloth until adult fruit fly or parasitoid emergence.

Botanical samples were identified by direct comparison with specimens from the herbarium of the Instituto de Ecología AC (INECOL)—XAL (Xalapa, Veracruz) and the Centro de Investigaciones de Yucatán (CICY) (Mérida, Yucatán). Adult fruit flies were identified by VHO (INECOL), while parasitoids 
were identified with the use of taxonomic keys [40] and with the help of Andrey Khalaim (Zoological Institute of the Russian Academy of Sciences, St. Petersburg, Russia). Updated information on scientific names of host plants was obtained by consulting the Tropicos database [41]. Reference specimens of identified plants were deposited in the XAL herbarium (INECOL). Reference samples of fruit flies were preserved in 70\% alcohol and deposited in INECOL and INBIOTECA, while parasitoid samples were deposited in INBIOTECA.

\subsection{Data Analyses}

Each sampled group of fruits of each species was weighted. For each sample, fruit infestation levels were calculated by dividing the total number of pupae obtained from the fruit sample by its total weight. The indexes of infestation by flies and of parasitism were obtained by dividing the total number of adult flies and/or parasitoids that emerged from the pupae by the total number of pupae obtained from the sample and multiplied by 100. All data from the localities were georeferenced, and coordinates were converted from degrees, minutes, and seconds (DMS) to decimal degrees (DD) using the website gps—coordinates.net. We used the DD to construct a transect map with GIS software (ArcMaps, Versión 10.6.1).

\section{Results}

\subsection{Fruit Fly-Host Plant Interactions}

We examined fruit samples from 76 plant species of 31 botanical families, which summed a total of $143.26 \mathrm{Kg}$. We documented the presence of 11 species of Anastrepha infesting 25 fruit species belonging to 10 families (Table 1).

Table 1. Host plant family, scientific and local names, and weight of sampled fruit found to be either, infested or uninfested by Anastrepha fruit flies during two annual cycles in natural areas of the Mayan rainforest of Quintana Roo, Mexico (March 2015-December 2017).

\begin{tabular}{|c|c|c|c|c|}
\hline Plant Family & Scientific Name & $\begin{array}{l}\text { Mayan Local } \\
\text { Name }\end{array}$ & $\begin{array}{c}\text { Sample } \\
\text { Weight } \\
\text { (Kg) }\end{array}$ & $\begin{array}{c}\text { Infested } \\
\text { Fruit } \\
\text { Yes/No }\end{array}$ \\
\hline \multirow{4}{*}{ Anacardiaceae } & Metopium brownei (Jacq.) & Chechen & 0.265 & $\mathrm{~N}$ \\
\hline & Spondias purpurea $\mathrm{L}$. & Ciruela & 1.100 & $\mathrm{Y}$ \\
\hline & Spondias mombin $\mathrm{L}$. & Jobo & 1.62 & $\mathrm{Y}$ \\
\hline & Mangifera indica L. & Mango & 4.58 & Y \\
\hline \multirow{3}{*}{ Annonaceae } & Annona globiflora Schlecht. & Anona & 0.270 & $\mathrm{~N}$ \\
\hline & Annona scleroderma Saff. & Chujun op & 0.680 & $\mathrm{~N}$ \\
\hline & Mosannona depressa (Baill.) Chatrou & Elemuy & 0.950 & $\mathrm{~N}$ \\
\hline \multirow{2}{*}{$\begin{array}{l}\text { Araliaceae } \\
\text { Bixaceae }\end{array}$} & Dendropanax arboreous (L.) Decne \& Planch. & Sakchaca & 0.1620 & $\mathrm{~N}$ \\
\hline & Cochlospermum vitifolium (Willd.) Spreng. & Chuun & 0.870 & $\mathrm{~N}$ \\
\hline \multirow{2}{*}{ Boraginaceae } & Ehretia tinifolia $\mathrm{L}$. & Beek & 0.282 & $\mathrm{~N}$ \\
\hline & Cordia dodecandra DC. & Ciricote & 5.310 & $\mathrm{~N}$ \\
\hline Burseraceae & Bursera simaruba (L.) Sarg. & Chaca Rojo & 0.176 & $\mathrm{~N}$ \\
\hline Cannabaceae & Celtis iguanaea (Jacq.) Sarg. & Muk & 0.475 & $\mathrm{~N}$ \\
\hline Capparaceae & Crataeva tapia $\mathrm{L}$. & Kookche & 3.320 & $\mathrm{~N}$ \\
\hline Caricaceae & Carica papaya L. & Chichput & 1.500 & $\mathrm{Y}$ \\
\hline Ebeneaceae & Diospyros anisandra S.F. Blake & Kabche & 0.300 & $\mathrm{~N}$ \\
\hline \multirow{2}{*}{ Euphorbiaceae } & Croton arboreus Millsp. & Perescuch & 0.310 & $\mathrm{~N}$ \\
\hline & Gymnanthes lucida Sw. & Yaiti & 1.200 & $\mathrm{~N}$ \\
\hline \multirow{7}{*}{ Fabaceae } & Caesalpinia platyloba S. Watson & Chacteviga & 0.235 & $\mathrm{~N}$ \\
\hline & Platymiscium yucatanum Standl. & Granadillo & 0.340 & $\mathrm{~N}$ \\
\hline & Piscidia piscipula (L.) Sarg. & Jabin & 0.410 & $\mathrm{~N}$ \\
\hline & Swartzia cubensis (Britton \& Wills) Standl. & Katalox & 0.790 & $\mathrm{~N}$ \\
\hline & Caesalpinia gaumeri (Britton \& Rose) Greenm. & Kitamche & 0.550 & $\mathrm{~N}$ \\
\hline & Lysiloma latisiliquum (L.) Benth. & Tzalam & 0.300 & $\mathrm{~N}$ \\
\hline & Lonchocarpus yucatanensis Pittier & Xuul & 0.260 & $\mathrm{~N}$ \\
\hline Lauraceae & Nectandra salicifolia (H.B.K.) Nees. & Sakelemuy & 1.650 & $\mathrm{~N}$ \\
\hline Malpighiaceae & Bunchosia swartziana Griseb. & Sipche & 0.615 & $\mathrm{~N}$ \\
\hline
\end{tabular}


Table 1. Cont.

\begin{tabular}{|c|c|c|c|c|}
\hline Plant Family & Scientific Name & $\begin{array}{l}\text { Mayan Local } \\
\text { Name }\end{array}$ & $\begin{array}{c}\text { Sample } \\
\text { Weight } \\
\text { (Kg) }\end{array}$ & $\begin{array}{c}\text { Infested } \\
\text { Fruit } \\
\text { Yes/No }\end{array}$ \\
\hline \multirow{4}{*}{ Malvaceae } & Hampea trilobata Standl. & Jool & 0.520 & $\mathrm{~N}$ \\
\hline & Luehea candida (DC.) Mart. & Kaskaat & 0.960 & $\mathrm{~N}$ \\
\hline & Pseudobombax ellipticum (Kunth) Dugand & Amapola & 0.700 & $\mathrm{~N}$ \\
\hline & Ceiba petandra (L.) Gaerth. & Yaaxche & 1.300 & $\mathrm{~N}$ \\
\hline \multirow{2}{*}{$\begin{array}{l}\text { Menispermaceae } \\
\text { Mimosaceae }\end{array}$} & Hyperbaena winzerlingii Standl. & Kekenche & 0.173 & $\mathrm{~N}$ \\
\hline & Acacia milleriana Standl. & Chimay & 0.150 & $\mathrm{~N}$ \\
\hline \multirow{2}{*}{ Moraceae } & Ficus pertusa L.f. & Juunkiix & 1.100 & $\mathrm{~N}$ \\
\hline & Brosimum alicastrum $\mathrm{Sw}$. & Ramon & 4.820 & $\mathrm{~N}$ \\
\hline \multirow{4}{*}{ Myrtaceae } & Psidium sartorianum (O. Berg) Nied. & Guayabillo & 0.885 & Y \\
\hline & Myrcianthes fragrans (Sw.) Mc Vaugh & Kojkann & 0.312 & $\mathrm{~N}$ \\
\hline & Eugenia biflora (L.) DC. & Pichiche & 0.500 & $\mathrm{~N}$ \\
\hline & Psidium guajava $\mathrm{L}$. & Guayaba & 1.225 & $\mathrm{Y}$ \\
\hline Opiliaceae & Agonandra macrocarpa L. O. Williams & Napche & 1.765 & $\mathrm{~N}$ \\
\hline \multirow{3}{*}{ Passifloraceae } & Passiflora foetida $\mathrm{L}$. & Poochil & 0.150 & $\mathrm{Y}$ \\
\hline & Passiflora serratifolia $\mathrm{L}$. & $\begin{array}{l}\text { Maracuya del } \\
\text { monte }\end{array}$ & 0.560 & Y \\
\hline & Passiflora yucatanensis Killip & Yaax pooch & 2.400 & Y \\
\hline Polygonaceae & Coccoloba acapulcensis Standl. & Boob/Toyub & 0.220 & $\mathrm{~N}$ \\
\hline Putranjivaceae & Drypetes lateriflora (Sw.) Krug \& Urb. & Ejuleb & 1.910 & $\mathrm{Y}$ \\
\hline Rhamnaceae & Krugiodendrom ferraum (Vahl) Urb. & Chintoc & 0.100 & $\mathrm{~N}$ \\
\hline \multirow{5}{*}{ Rubiaceae } & Cosmocalyx spectabilis Standl. & Chactecook & 0.164 & $\mathrm{~N}$ \\
\hline & Randia truncata Greenm. \& C.H.Thomps. & Kaakalche & 0.400 & $\mathrm{~N}$ \\
\hline & Exostema mexicanum A Gray & Sabasche & 0.395 & $\mathrm{~N}$ \\
\hline & Guettarda combsii Urb. & Tastab & 0.270 & $\mathrm{~N}$ \\
\hline & Morinda citrifolia $\mathrm{L}$. & Noni & 3.500 & $\mathrm{~N}$ \\
\hline \multirow{4}{*}{ Rutaceae } & Citrus aurantium $\mathrm{L}$. & Naranja agria & 6.270 & $\mathrm{Y}$ \\
\hline & Esenbeckia pentaphylla (Macfad.) Griseb. & Narnaha che & 2.630 & $\mathrm{~N}$ \\
\hline & Citrus sinensis (L.) Osbek & Naranja dulce & 3.310 & $\mathrm{Y}$ \\
\hline & Casimiroa microcarpa Lundell & Yuuy & 7.300 & $\mathrm{Y}$ \\
\hline \multirow{3}{*}{ Salicaceae } & Laetia thamnia L. & Chauche & 3.141 & $\mathrm{Y}$ \\
\hline & Casearia corymbosa Kunth & Ixiimche & 0.424 & $\mathrm{~N}$ \\
\hline & Zuelania guidonia (Sw.) Britton \& Millsp. & Tamay & 5.672 & $\mathrm{Y}$ \\
\hline \multirow{7}{*}{ Sapindaceae } & Blomia prisca (Standl.) Lundell & Tzol & 4.900 & $\mathrm{Y}$ \\
\hline & Cupania belizensis Standl. & Sal poom & 1.200 & $\mathrm{~N}$ \\
\hline & Thouinia paucidentata Radlk. & Kanchunup & 0.136 & $\mathrm{~N}$ \\
\hline & Melicoccus bijugatus Jacq. & Guaya & 1.200 & $\mathrm{~N}$ \\
\hline & Matayba oppositifolia (A. Rich.) Britton & Ikche & 0.370 & $\mathrm{~N}$ \\
\hline & Allophylus camptostachys Radlk. & Kanchunup & 0.783 & $\mathrm{~N}$ \\
\hline & Talisia oliviformis (Kunth) Radlk. & Wayum & 1.380 & $\mathrm{~N}$ \\
\hline \multirow{9}{*}{ Sapotaceae } & Manilkara zapota (L.) Van Royen & Chicozapote & 4.200 & $\mathrm{Y}$ \\
\hline & Chrysophyllum cainito L. & Cayumito & 2.800 & Y \\
\hline & $\begin{array}{l}\text { Chrysophyllum mexicanum Brandegee ex } \\
\text { Standl. }\end{array}$ & Chique & 0.690 & Y \\
\hline & Pouteria campechiana (Kunth) Baehni & Kaniste & 9.393 & Y \\
\hline & Pouteria sapota (Jacq.) H. E. Moore and Stearn & Hazz & 3.500 & Y \\
\hline & $\begin{array}{l}\text { Sideroxylon capiri subsp. tempisque (Pittier) } \\
\text { T.D. Penn. }\end{array}$ & Subul & 6.554 & $\mathrm{Y}$ \\
\hline & $\begin{array}{l}\text { Sideroxylon foetidissimum subsp. gaumeri } \\
\text { (Pittier) T.D. Penn. }\end{array}$ & Tsiimimche & 0.800 & $\mathrm{~N}$ \\
\hline & Pouteria glomerata (Miq.) Radlk. & $\begin{array}{l}\text { Zapote del } \\
\text { pueblo }\end{array}$ & 9.935 & Y \\
\hline & Pouteria reticulata (Engl.) Eyma & Zapotillo & 5.220 & Y \\
\hline Simaroubaceae & Simarouba glauca DC. & Paasac & 0.360 & $\mathrm{~N}$ \\
\hline Verbenaceae & Vitex gaumeri Greenm. & Yaxnic & 9.096 & Y \\
\hline
\end{tabular}

Our sampling efforts resulted in the first records of 10 plant species and a plant family (Putranjivaceae) serving as new hosts of fruit flies of the genus Anastrepha. These plant species are: Drypetes lateriflora (Sw.) Krug and Urb. (Putranjivaceae) and Blomia prisca (Standl.) Lundell (Sapindaceae), hosts for Anastrepha fraterculus (Wiedemann); Passiflora yucatanensis Killip Passiflora serratifolia L., and Passiflora foetida L. (Passifloraceae) infested by Anastrepha chiclayae Greene; Laetia thamnia L. (Salicaceae), infested by Anastrepha zuelaniae Stone; Pouteria reticulata (Engl.) Eyma 
(Sapotaceae), infested by Anastrepha serpentina (Wiedemann) and Anastrepha. sp. (new species); Vitex gaumeri Greenm. (Verbenaceae), infested by Anastrepha ampliata Hernández-Ortiz (Figure 2B), and recently cited for Campeche [42]; and Casimiroa microcarpa Lundell (Rutaceae) infested by Anastrepha ludens (Loew) (Table 2, Figure 3A-D).

Of all the fruit fly species, A. serpentina exhibited the highest number of hosts in the region, exploiting up to eight host species, all in the family Sapotaceae. The Mexican fruit fly, A. ludens, was found in three species of plants of the family Rutaceae and the West Indian fruit fly, Anastrepha obliqua (Macquart), was found in three species of the family Anacardiaceae. The guava fruit fly, Anastrepha striata Schiner and Anastrepha curvicauda (Gerstaecker) were found in single host plant species: Psidium guajava L. (Myrtaceae) and Carica papaya L. (Caricaceae), respectively (Table 2).

With respect to host plant phenology, the highest availability of fruits was generally observed in the period of April-July, with the highest number and abundance of fruits recorded during May (18 species). In particular, we observed that the fruits of M. zapota were present during the whole annual cycle, whereas the two species of the family Salicaceae, which were hosts for A. zuelaniae, showed the shortest fructification periods (Table 2).
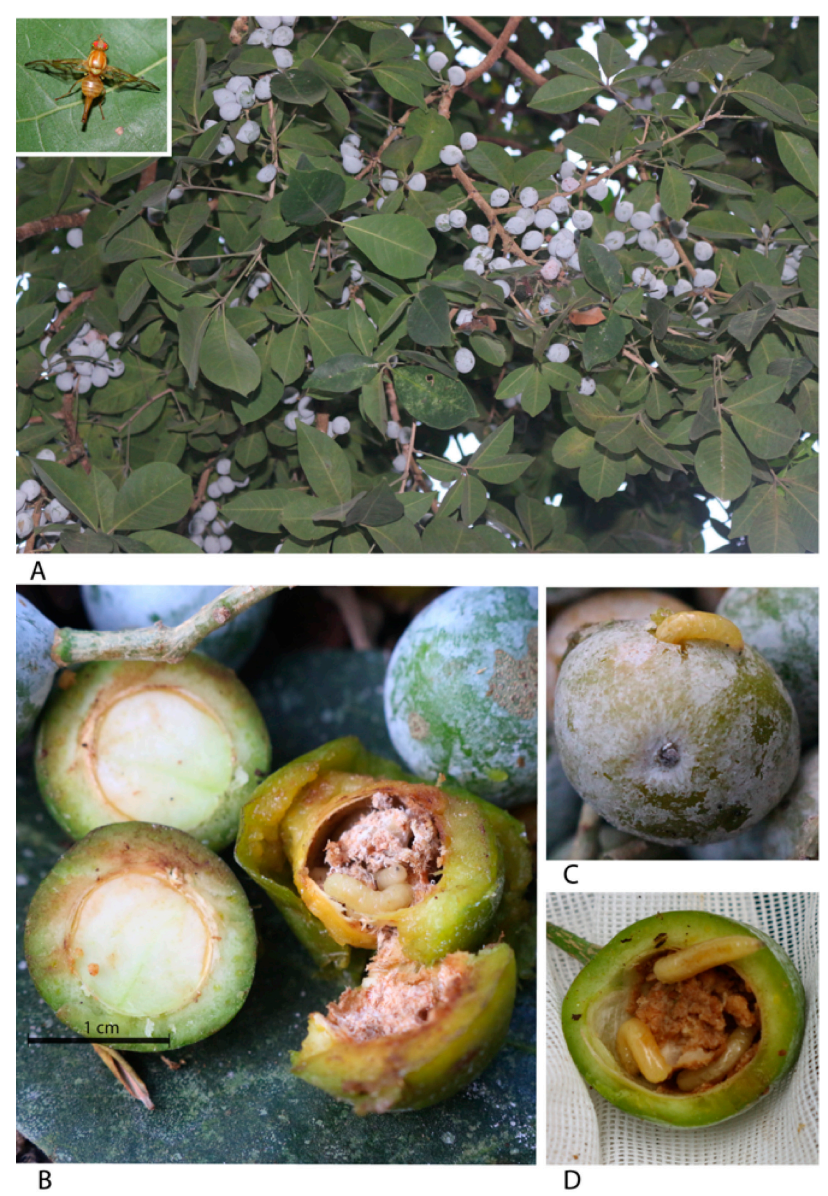

Figure 3. (A) Host plant Casimiroa microcarpa (first report) with fruits, (B) fruits with seeds with and without infestation, (C) Larvae of $A$. ludens emerging from the fruits, (D) Larvae of $A$. ludens feeding on the seed of C. microcarpa. 
Table 2. Distribution of the fructification period of plant species sampled from natural areas of the Mayan rainforest of Quintana Roo, Mexico (March 2015-December 2017). Darker shading indicates greater availability of fruits; lighter shading denotes a decreased fruit availability, generally occurring before and after the rainy season. Asterisks indicate new host plant records for Anastrepha spp.

\begin{tabular}{|c|c|c|c|c|c|c|c|c|c|c|c|c|c|c|}
\hline Host Family & Host Scientific Name & Fruit Fly Species & Jan & Feb & Mar & Apr & May & Jun & Jul & Aug & Sep & Oct & Nov & Dec \\
\hline \multirow[t]{2}{*}{ Anacardiaceae } & $\begin{array}{l}\text { Mangifera indica } \mathrm{L} \text {. } \\
\text { Spondias purpurea } \mathrm{L} \text {. }\end{array}$ & $\begin{array}{l}\text { A. obliqua } \\
\text { A. obliqua }\end{array}$ & & & & & & & & & & & & \\
\hline & Spondias mombin $\mathrm{L}$. & A. obliqua & & & & & & & & & . & . & . & \\
\hline Caricaceae & Carica papaya $\mathrm{L}$. & A. curvicauda & & & , & & & & & & & & & \\
\hline \multirow[t]{3}{*}{ Myrtaceae } & Psidium guajava L. & A. striata & & & & & & & & . & . & . & & \\
\hline & Psidium guajava & A. fraterculus & & & 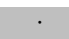 & & & & & & & & & \\
\hline & Psidium sartorianum (O. Berg) Nied. & A. fraterculus & & & . & & & & & & & & & \\
\hline Putranjivaceae & ${ }^{*}$ Drypetes lateriflora (Sw.) Krug \& Urb. & A. fraterculus & & . & . & . & & & & & & & & \\
\hline Sapindaceae & *Blomia prisca (Standl.) Lundell & A. fraterculus & & & & & & & & & & & & \\
\hline \multirow[t]{3}{*}{ Passifloraceae } & *Passiflora yucatanensis Killip & A. chiclayae & & . & . & r. & & & & & & & & \\
\hline & ${ }^{*}$ Passiflora serratifolia L. & A. chiclayae & & & . & & & & & & & & & \\
\hline & *Passiflora foetida $\mathrm{L}$. & A. chiclayae & & & & & & & & . & . & & & \\
\hline \multirow[t]{3}{*}{ Rutaceae } & Citrus aurantium $\mathrm{L}$. & A. ludens & . & . & . & & & & & & & . & & \\
\hline & Citrus sinensis (L.) Osbek & A. ludens & . & . & & & & & & & & & & \\
\hline & ${ }^{*}$ Casimiroa microcarpa Lundell & A. ludens & & & . & & & & & & & & & \\
\hline \multirow[t]{2}{*}{ Salicaceae } & ${ }^{*}$ Laetia thamnia $\mathrm{L}$. & A. zuelaniae & & & & & & & & . & . & & & \\
\hline & Zuelania guidonia (Sw.) Britton \& Millsp. & A. zuelaniae & & & & & & & & . & & & & \\
\hline \multirow[t]{10}{*}{ Sapotaceae } & Chrysophyllum mexicanum Brandegee ex Standl. & A. serpentina & . & . & . & & & & & & & & & \\
\hline & Chrysophyllum cainito $\mathrm{L}$. & A. serpentina & & & . & & & & & & & & & \\
\hline & Manilkara zapota (L.) Van Royen & A. serpentina & . & . & . & & & & & . & . & . & . & \\
\hline & Pouteria campechiana Baehni & A. serpentina & & & . & & & & & . & . & . & . & \\
\hline & Pouteria glomerata (Miq.) Radlk. & A. serpentina & & & & & & & & . & . & . & . & \\
\hline & *Pouteria reticulata (Engl.) Eyma & A. serpentina & & & & . & & & & & & . & . & \\
\hline & Pouteria sapota (Jacq.) H.E. Moore and Stearn. & A. serpentina & & & & & & & & & . & & & \\
\hline & Sideroxylon capiri subsp. tempisque (Pittier) T.D. Penn. & A. serpentina & & & & & & & & & & & & \\
\hline & Pouteria campechiana Baehni & A. hamata & & & . & & & & & . & . & . & e & \\
\hline & ${ }^{*}$ Pouteria reticulata & Anastrepha sp. 1 & & & & & & & & & & . & . & \\
\hline Verbenaceae & *Vitex gaumeri Greenm. & A. ampliata & & & & & & & & . & . & . & . & \\
\hline
\end{tabular}




\subsection{Fruit Infestation and Parasitism Rates}

Fruit infestation rates were highly variable between the different hosts, ranging between 0.21 and 19.17 pupae/kg of sampled fruit. The highest infestation levels occurred in Spondias mombin L. B. prisca, P. reticulata, and Sideroxylon capiri subsp tempisque (Pittier) T.D. Penn. (range: 14.6-19.1 pupae/kg fruit). Of these, $P$. reticulata and $S$. capiri showed the highest infestation index values in relation to the size of the sample, compared to other Sapotaceae species of the genus Pouteria who had only $1.2-2.0 \mathrm{pupae} / \mathrm{kg}$ fruit. The highest fly emergence value was observed in P. campechana Baehni, with $97.44 \%$ of the flies emerging from pupae, and the lowest rates were observed in S. mombin (47.86\%) and P. reticulata (47.83\%) (see Table 3).

Table 3. Fruit fly species of the genus Anastrepha, and their infestation levels and biological data, found in plant species associated as their native and introduced hosts in the sampled region.

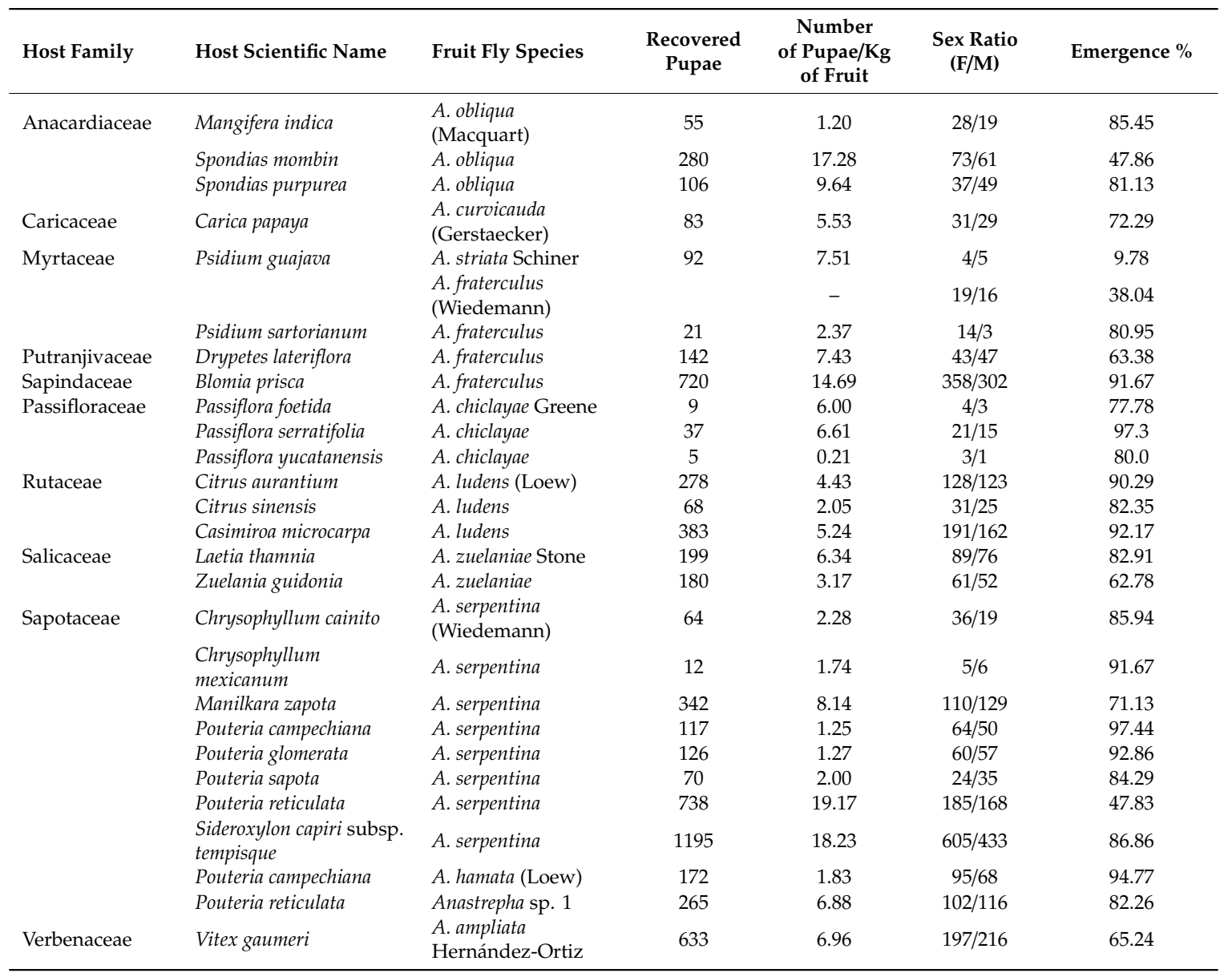

We recorded an overall parasitism rate of $19.51 \%$, which means that, at the community level, the fly/parasitoid ratio was 5:1. We recorded six species of koinobiont parasitoids: Doryctobracon areolatus (Szépligeti), Doryctobracon zeteki Musebeck, Utetes anastrephae (Viereck), Opius hirtus (Fisher) (all Braconidae), Aganaspis pelleranoi (Brethés), and Odontosema anastrephae Borgmeier (both Figitidae). All these species parasitized larvae that feed on the pulp of the fruit, with the exception of $D$. areolatus and D. zeteki, which also parasitized larvae that infest seeds. Doryctobracon areolatus was the most important parasitoid species in the whole community, representing $52.7 \%$ of all the recorded parasitoids in terms of abundance. It was present in 12 different species of host plants, parasitizing six species of fruit flies.

The percentage of parasitism fluctuated between $3.49 \%$ (for A. hamata (Loew) feeding on P. campechiana) and $35.54 \%$, (for A. serpentina feeding on $P$. reticulata). In the case of the species 
A. ludens and $A$. chiclayae, we did not observe any parasitism. Other species, such as $U$. anastrephae, A. pelleranoi, and $O$. hirtus, showed moderate activity, with parasitism rates ranging between 13.8 and $17.8 \%$. The least frequently recovered parasitoid species were O. anastrephae and D. zeteki, with 0.6 and $0.7 \%$ of parasitism, respectively (Table 4 ). The fly species with the highest richness of parasitoids were $A$. fraterculus and $A$. serpentina, with five species each; in contrast, A. hamata and $A$. sp. had only one species each, independently of the number of hosts occupied.

Table 4. Parasitoid species and levels of parasitism of fruit fly species of the genus Anastrepha in their native and introduced hosts in the sampled region.

\begin{tabular}{|c|c|c|c|c|c|c|c|}
\hline Family & $\begin{array}{c}\text { Host Plant } \\
\text { Scientific Name }\end{array}$ & $\begin{array}{l}\text { Anastrepha } \\
\text { Species }\end{array}$ & $\begin{array}{c}\text { Recovered } \\
\text { Fruit Fly } \\
\text { Pupae }\end{array}$ & $\begin{array}{l}\text { Parasitoid } \\
\text { Species }\end{array}$ & $\begin{array}{l}\text { Parasitoid } \\
\text { Sex Ratio } \\
\text { (F/M) }\end{array}$ & $\begin{array}{l}\text { Total No. } \\
\text { Parasitoids }\end{array}$ & $\begin{array}{c}\% \\
\text { Parasitism }\end{array}$ \\
\hline \multirow[t]{4}{*}{ Anacardiaceae } & Mangifera indica & A. obliqua & 55 & $\begin{array}{l}\text { Doryctobracon } \\
\text { areolatus } \\
\text { (Szépligeti) }\end{array}$ & $1 / 2$ & 3 & 5.45 \\
\hline & Spondias mombin & & 280 & $\begin{array}{l}\text { D. areolatus } \\
\text { Utetes }\end{array}$ & $38 / 25$ & & \\
\hline & & & & $\begin{array}{l}\text { anastrephae } \\
\text { (Viereck) }\end{array}$ & $15 / 21$ & 99 & 35.36 \\
\hline & Spondias purpurea & & 106 & D. areolatus & $10 / 8$ & 18 & 16.98 \\
\hline \multirow[t]{3}{*}{ Myrtaceae } & Psidium guajava & A. fraterculus & 92 & D. areolatus & $8 / 5$ & & \\
\hline & & & & $\begin{array}{l}\text { pelleranoi } \\
\text { (Brethes) }\end{array}$ & $7 / 2$ & & \\
\hline & & & & $\begin{array}{l}\text { Odontosema } \\
\text { anastrephae } \\
\text { Borgmeier }\end{array}$ & $2 / 1$ & 25 & 27.17 \\
\hline \multirow[t]{4}{*}{ Putranjivaceae } & Drypetes lateriflora & & 142 & D. areolatus & $5 / 3$ & & \\
\hline & & & & U. anastrephae & $3 / 4$ & & \\
\hline & & & & $\begin{array}{l}\text { Opius hirtus } \\
\text { (Fisher) }\end{array}$ & $2 / 1$ & & \\
\hline & & & & A. pelleranoi & $8 / 12$ & 38 & 26.76 \\
\hline \multirow[t]{3}{*}{ Sapindaceae } & Blomia prisca & & 720 & D. areolatus & $27 / 30$ & & \\
\hline & & & & U. anastrephae & $12 / 11$ & & \\
\hline & & & & O. hirtus & $7 / 4$ & 91 & 12.64 \\
\hline \multirow{4}{*}{ Salicaceae } & Laetia thamnia & A. zuelaniae & 199 & D. areolatus & $13 / 17$ & & \\
\hline & & & & O. hirtus & $2 / 0$ & 32 & 16.08 \\
\hline & Zuelania guidonia & & 180 & D. areolatus & $12 / 7$ & & \\
\hline & & & & A. pelleranoi & $4 / 3$ & 26 & 14.44 \\
\hline \multirow[t]{11}{*}{ Sapotaceae } & Manilkara zapota & A. serpentina & 342 & D. areolatus & $16 / 17$ & & \\
\hline & & & & O. hirtus & $4 / 2$ & & \\
\hline & & & & A. pelleranoi & $22 / 15$ & & \\
\hline & & & & O. anastrephae & $3 / 1$ & 80 & 23.81 \\
\hline & Pouteria reticulata & & 738 & D. areolatus & $29 / 31$ & & \\
\hline & & & & U. anastrephae & $48 / 38$ & & \\
\hline & & & & O. hirtus & $28 / 21$ & & \\
\hline & & & & A. pelleranoi & $37 / 21$ & 263 & 35.54 \\
\hline & Sideroxylon capiri su & sp. tempisque & 1195 & D. areolatus & $35 / 39$ & & \\
\hline & & & & O. hirtus & $11 / 4$ & & \\
\hline & & & & A. pelleranoi & $1 / 2$ & 112 & 9.37 \\
\hline \multirow[t]{2}{*}{ Sapotaceae } & $\begin{array}{l}\text { Pouteria } \\
\text { campechiana }\end{array}$ & A. hamata & 172 & $\begin{array}{l}\text { Doryctobracon } \\
\text { zeteki } \\
\text { Musebeck }\end{array}$ & $5 / 1$ & 6 & 3.49 \\
\hline & Pouteria reticulata & Anastrepha sp. & 265 & D. areolatus & $7 / 12$ & & 7.17 \\
\hline \multirow[t]{3}{*}{ Verbenaceae } & Vitex gaumeri & A. ampliata & 633 & D. areolatus & $58 / 51$ & & \\
\hline & & & & U. anastrephae & $15 / 11$ & & \\
\hline & & & & O. hirtus & $27 / 25$ & 187 & 29.54 \\
\hline
\end{tabular}

\section{Discussion}

Previous studies reported 39 known described species of Anastrepha in Mexico [24,43], including recent records of Anastrepha tehuacana Norrbom [16] and Anastrepha furcata Lima [44]. For the state of Quintana Roo, there are currently 12 known species of Anastrepha [24,36,43,45,46]. The present study contributes with the first records of four additional species: Anastrepha chiclayae Greene, Anastrepha zuelaniae Stone, Anastrepha curvicauda, and a newly discovered species, Anastrepha sp., increasing the number of known Anastrepha species for the state of Quintana Roo to 16. 
In addition, here we document the first records of eight new hosts plants for fruit flies, including the family Putranjivaceae for the first time. Furthermore, 15 hosts are reported for the first time in Quintana Roo. These results highlight the importance of increasing our knowledge about fruit fly/host plant interactions in natural environments. The Mayan rainforest in the southeast of Mexico constitutes a reservoir for tropical biodiversity, including interactions between fruit flies and their natural enemies. Even though the traditional use of protein-baited traps is important to provide data on the presence and abundance of Anastrepha species in a particular region, species richness is higher for native fruits [47].

A noteworthy result is the exploitation of alternative hosts by species of economic significance such as A. ludens. In Mexico, this fly species has been reported feeding on approximately 23 host plants, most of them being exotic cultivated species, such as Citrus spp. [24]. Two important native host plant species are Casimiroa greggii (S. Watson) F. Chiang and Casimiroa edulis Llave et Lex [48,49] (both Rutaceae). In the present study, A. ludens was recovered from fruits of two Citrus species and from Casimiroa microcarpa Lundell. The latter plant species is a new record for A. ludens in Quintana Roo, which was thought to be restricted to Chiapas and Guatemala [50]. In C. microcarpa, the larvae were found to feed exclusively on the seeds (Figure 3), as previously observed in C. greggii [48,51]. These habits suggest that the use of these native wild hosts could have broadened the distribution area of these flies through the colonization of citrus species cultivated in other regions of Mexico, while at the same time retaining their native hosts of the genus Casimiroa because of their distribution in the region. For example, C. greggii is found in the northeast of Mexico, C. edulis is distributed from Mexico to Costa Rica, and C. microcarpa is distributed mainly in Guatemala [41].

The center of origin of the family Sapotaceae is tropical America, and plant species belonging to this family are of great importance in the structure of ecosystems and biological diversity with approximately 200 genera and close to 450 species of trees and shrubs [52,53]. In addition, the consumption of their fruits represents a highly profitable market. For example, Manilkara zapota L. is native to Yucatán (Mexico) and Guatemala [54], and its fruits, which have high commercial value, occur practically all year round are commonly heavily infested by A. serpentina. So these are also significant reservoirs of native parasitoids.

Fruit flies can persist in different types of environments. Generalist species can thrive in a matrix of human use with commercial and backyard fruit orchards, while a part of the population remains and survives within the natural forest. That would be the case of $A$. ludens in C. microcarpa, a plant species that maintains viable populations of this fruit fly within their natural habitat. Because 70 percent of herbivore species exhibit a high level of specialization, [55], then knowledge of wild plant species that serve as hosts for specialist fruit flies is relevant. For example, A. zuelaniae, A. ampliata, A. chiclayae, A. hamata, and $A$. sp., have a restricted range of plants (families Salicaceae, Verbenaceae, Passifloraceae, and Sapotaceae, respectively) on which they feed. An interesting observation was that the fruits of Pouteria glomerata (Miq.) Radlk (Sapotaceae) were only infested by A. serpentina in the study area, even though fruits of this plant species have been found to be infested by Anastrepha aphelocentema Stone [37]. The absence of the latter species may implicate biogeographic and ecological factors that could be responsible for the presence/absence of certain species in a particular site [56,57].

The high percentages of parasitism observed in this study in hosts, such as Pouteria reticulata (Engl.) Eyma, Spondias mombin L., and Vitex gaumeri Greenm (29.5-35.6\%), differ from previous reports for Yucatán that show that parasitism levels are low. For example, Hernández-Ortiz et al. [26] documented an overall parasitism rate of $3.69 \%$ from cultivated plants. Such contrasting results suggest that the Mayan rainforest actually constitutes a highly important reservoir for the diversity of native parasitoid species. This study confirms that Doryctobracon areolatus (Szépligeti) is the native parasitoid with the highest abundance. This parasitoid species is widely found in Mexico and other countries [31,33,34]. Moreover, we report Opius hirtus (Fisher) in five new fruit fly-parasitoid associations, all occurring in native tree species infested by different fly species. This finding highlights the preference of this parasitoid for monophagous fly species attacking comparatively small-sized fruits [19,31]. 
The presence of the parasitoid Doryctobracon zeteki Muesebeck in larvae of $A$. hamata infesting P. campechiana shows the occurrence of a parasitoid attacking larvae of a fly species that feeds on seeds. This is the first report of parasitism in A. hamata. In the case of D. zeteki, this parasitoid species was first recorded in Mexico in association with larvae of Anastrepha cordata Aldrich feeding on seeds [19]. However, in countries like Colombia, Costa Rica, Panama, and Venezuela, D. zeteki has been reported in P. guajava [34], in Sapotaceae species, and in other species where it has been recorded as the most abundant species $[25,58]$. Interestingly, some fruit fly species, such as A. serpentina, infesting Pouteria sapota (Jacq.) H.E. Moore and Stearn and P. glomerata, and A. chiclayae, infesting passion flowers, did not exhibit parasitism in their natural hosts, which could be a result of the large size of the fruits they infest. This could be a defense mechanism, since it would be more difficult for parasitoids to find a host inside large fruits, which has been hypothesized for other frugivorous species of Anastrepha [31,59,60].

The exotic parasitoid Diachasmimorpha longicaudata (Ashmead) has been successfully established in certain tropical agroecosystems with significant percentages of parasitism [34,61-63]. However, the results of the present study showed that this species is not established in this natural region of the Mayan rainforest, and therefore, this introduced species appears to have limitations for its establishment in natural environments $[19,26,31]$. For example, we did not find it in fruits of Citrus spp. infested by A. ludens, where it is common in other regions, along with the native species Doryctobracon crawfordi (Viereck) [33].

\section{Conclusions}

Our findings shed new light into new host plant association for species of the genus Anastrepha and their parasitoids in natural environments, and highlight the importance of tropical rainforests for the conservation of biodiversity. The areas of the Mayan rainforest that still preserve a great part of its original composition and structure exhibit a higher richness of wild fruits, such as those examined in this study. Consequently, this represent a highly important reservoir for the diversity of native parasitoids spatially and temporally that are practically absent in fruits of cultivated plants.

Author Contributions: Conceptualization, M.L.-O., V.H.-O.; Formal analysis, M.L.-O.; Funding acquisition, M.L.-O.; Investigation, M.L.-O., J.R.V.-L., M.H.-O.; Methods, M.L.-O., J.R.V.-L., M.H.-O.; Supervision, V.H.-O., F.D.-F., J.C.P.; Writing-original draft, M.L.-O., V.H.-O.; Writing-review and editing, V.H.-O., F.D.-F., J.C.P. All authors have read and agreed to the published version of the manuscript.

Funding: This study was partially supported by Programa de Mejoramiento del Profesorado (PROMEP) (grant number P/PROMEP/103.5/13/6998), awarded to M.L.-O., and by the Consejo Nacional de Ciencia y Tecnología-Sistema Nacional de Investigadores de México (CONACyT-SNI 59909 MLO). The funders had no role in the collection, analyses, or interpretation of the data, in the writing of the manuscript, or in the decision to publish the results.

Acknowledgments: We are grateful to Carlos Duran (INECOL), Rodrigo Duno de Stefano (CICY), and Andrey Khalain (Zoological Institute, St. Petersburg, Russia) for the identification of field-collected material. We also thank the inhabitants of Dzula, municipality of Carrillo Puerto, for access to their natural areas, especially to Artemio Xiu Tamayo for his help in the collection of fruits in the field in order to obtain Anastrepha spp adults, and the Xiu family for their hospitality. We also thank Rubén Ruiz (La selva) and Roy Jabin Carreón (UNORCA) for vehicle access, and Daniel Gazga (SEMAR) for his logistical support. Thanks to Fernando Díaz, Ernesto Cervantes, Roberto Bautista (INBIOTECA) and Hugo Ortiz for technical assistance. Three anonymous reviewers greatly improved an early version of the manuscript.

Conflicts of Interest: The authors declare no conflict of interest.

\section{References}

1. Becerra, J.X. On the factors that promote the diversity of herbivorous insects and plants in tropical forests. Proc. Natl. Acad. Sci. USA 2015, 112, 6098-6103. [CrossRef] [PubMed]

2. Price, P.W.; Denno, R.F.; Eubanks, M.D.; Finke, D.L.; Kaplan, I. Insect Ecology: Behavior, Populations and Communities; Cambridge University Press: Cambridge, UK, 2011.

3. Keck, C.B. Relation of oviposition punctures of the Mediterranean fruit fly to the premature dropping of citrus fruits. J. Econ. Entomol. 1934, 28, 908-914. [CrossRef] 
4. Stephenson, A.G. Flower and fruit abortion: Proximate causes and ultimate functions. Annu. Rev. Ecol. Syst. 1981, 12, 253-279. [CrossRef]

5. Drew, R.A.I. Amino acid increases in fruit infested by fruit flies of the family Tephritidae. Zool. J. Linn. Soc. 1988, 93, 107-112. [CrossRef]

6. Pimm, S.; Russell, G.; Gittleman, J.; Brooks, T. The future of Biodiversity. Science 1995, 269, 347-350. [CrossRef]

7. Fahrig, L. Effects of habitat fragmentation on biodiversity. Annu. Rev. Ecol. Syst. 2003, 34, 487-515. [CrossRef]

8. Norrbom, A.L.; Korytkowski, C.A.; Zucchi, R.A.; Uramoto, K.; Venable, G.L.; McCormick, J.; Dallwitz, M.J. Anastrepha and Toxotrypana: Descriptions, Illustrations, and Interactive Keys; Version: 16th October 2018; 2012; Dallwitz, Paine, and Zurcher 1995, 2000, USDA. Available online: http://delta-intkey.com (accessed on 6 December 2018).

9. Eskafi, F.M.; Cunningham, R.T. Host plants of fruit flies (Diptera: Tephritidae) of economic importance in Guatemala. Fla. Entomol. 1987, 70, 116-123. [CrossRef]

10. Jiron, L.F.; Hedström, I. Occurrence of fruit flies of the genera Anastrepha and Ceratitis (Diptera: Tephritidae), and their host plant availability in Costa Rica. Fla. Entomol. 1988, 71, 62-73. [CrossRef]

11. Borge, M.N.R.; Basedow, T. A survey on the occurrence and flight periods of fruit fly species (Diptera: Tephritidae) in a fruit growing area in southwest Nicaragua, 1994/95. Bull. Entomol. Res. 1997, 87, 405-412. [CrossRef]

12. Uramoto, K.; Martins, D.S.; Zucchi, R.A. Fruit flies (Diptera, Tephritidae) and their associations with native host plants in a remnant area of the highly endangered Atlantic Rain Forest in the State of Espírito Santo, Brazil. Bull. Entomol. Res. 2008, 98, 457-466. [CrossRef]

13. Silva, J.G.; Dutra, V.S.; Santos, M.S.; Silva, N.M.; Vidal, D.B.; Nink, R.A.; Guimaraes, J.A.; Araujo, E.L. Diversity of Anastrepha spp. (Diptera: Tephritidae) and associated braconid parasitoids from native and exotic hosts in Southeastern Bahia, Brazil. Environ. Entomol. 2010, 39, 1457-1465. [CrossRef] [PubMed]

14. Garcia, F.R.; Norrbom, A.L. Tephritoid flies (Diptera, Tephritoidea) and their plant hosts from the state of Santa Catarina in southern Brazil. Fla. Entomol. 2011, 94, 151-157. [CrossRef]

15. Jesus-Barros, C.R.; Adaime, R.; Oliveira, M.N.; Silva, W.R.; Costa-Neto, S.V.; Souza-Filho, M.F. Anastrepha (Diptera: Tephritidae) species, their hosts and parasitoids (Hymenoptera: Braconidae) in five municipalities of the state of Amapá, Brazil. Fla. Entomol. 2012, 95, 694-705. [CrossRef]

16. Norrbom, A.L.; Castillo-Meza, A.L.; García-Chávez, J.H.; Aluja, M.; Rull, J. A new species of Anastrepha (Diptera: Tephritidae) from Euphorbia tehuacana (Euphorbiaceae) in Mexico. Zootaxa 2014, 3780, 567-576. [CrossRef]

17. Clavijo, P.A.R.; Norrbom, A.L.; Peñaranda, E.A.; Diaz, P.A.; Benitez, M.C.; Gallego, J.; Cruz, M.I.; Montes, J.M.; Rodriguez, E.J.; Steck, G.J.; et al. New records of Anastrepha (Diptera: Tephritidae) primarily from Colombia. Zootaxa 2018, 4390, 1-63. [CrossRef] [PubMed]

18. Hernandez-Ortiz, V.; Pérez-Alonso, R. The natural host plants of Anastrepha (Diptera: Tephritidae) in a tropical rain forest of Mexico. Fla. Entomol. 1993, 70, 447-460. [CrossRef]

19. Aluja, M.; Rull, J.; Sivinski, J.; Norrbom, A.L.; Wharton, R.A.; Macías-Ordóñez, R.; Díaz-Fleischer, F.; López, M. Fruit flies of the genus Anastrepha (Diptera: Tephritidae) and associated native parasitoids (Hymenoptera) in the tropical rainforest biosphere reserve of Montes Azules, Chiapas, Mexico. Environ. Entomol. 2003, 32, 1377-1385. [CrossRef]

20. Deus, E.G.; Pinheiro, L.S.; Lima, C.R.; Sousa, M.D.S.M.; Guimarāes, J.A.; Strikis, P.C.; Adaime, R. Wild hosts of frugivorous dipterans (Tephritidae and Lonchaeidae) and associated parasitoids in the Brazilian Amazon. Fla. Entomol. 2013, 96, 1621-1625. [CrossRef]

21. Almeida, R.R.; Cruz, K.R.; Sousa, M.S.M.; Costa-Neto, S.V.; Jesus Barros, C.R.; Lima, A.L.; Adaime, R. Frugivorous flies (Diptera: Tephritidae, Lonchaeidae) associated with fruit production on Ilha de Santana, Brazilian Amazon. Fla. Entomol. 2016, 99, 426-436. [CrossRef]

22. Hernández-Ortiz, V.; Aluja, M. Listado de especies del género neotropical Anastrepha (Diptera: Tephritidae), con notas sobre su distribución y plantas hospederas. Folia Entomol. Mex. 1993, 88, 89-105.

23. Hernández-Ortiz, V.; Canal, N.A.; Salas, J.O.T.; Ruíz-Hurtado, F.M.; Dzul-Cauich, J.F. Taxonomy and phenotypic relationships of the Anastrepha fraterculus complex in the Mesoamerican and Pacific Neotropical dominions (Diptera, Tephritidae). ZooKeys 2015, 540, 95-124. [CrossRef] [PubMed] 
24. Hernández-Ortiz, V. Diversidad y biogeografía del género Anastrepha en México. In Moscas de la Fruta en Latinoamérica (Diptera: Tephritidae): Diversidad, Biología y Manejo; S y G, Ed.; Distrito Federal, Mexico, 2007; pp. 53-76.

25. Carrejo, N.S.; Gonzalez, R. Parasitoids reared from species of Anastrepha (Diptera: Tephritidae) in Valle del Cauca, Colombia. Fla. Entomol. 1999, 82, 113-118. [CrossRef]

26. Hernández-Ortiz, V.; Delfín-González, H.; Escalante-Tio, A.; Manrique-Saide, P. Hymenopteran parasitoids of Anastrepha fruit flies (Diptera: Tephritidae) reared from different hosts in Yucatan, Mexico. Fla. Entomol. 2006, 89, 508-515. [CrossRef]

27. Clausen, C.P.; Clancy, D.W.; Chock, Q.C. Biological Control of the Oriental Fruit Fly (Dacus dorsalis Hendel) and Other Fruit Flies in Hawaii; USDA Tech. Bull.: Washington, DC, USA, 1965; Volume 1322, p. 102.

28. Wharton, R.A. Classical Biological Control of Fruit-Infesting Tephritidae. In World Crop Pests, Fruit Flies: Their Biology, Natural Enemies and Control; Robinson, A.S., Hooper, G., Eds.; Elsevier: Amsterdam, The Netherlands, 1989; Volume 3B, pp. 303-313.

29. Leonel, F.L., Jr.; Zucchi, R.A.; Wharton, R.A. Distribution and tephritid hosts (Diptera) of braconid parasitoids (Hymenoptera) in Brazil. Int. J. Pest Manag. 1995, 41, 208-213. [CrossRef]

30. Wharton, R.A.; Gilstrap, F.E.; Rhode, R.H.; Fischel-m, M.; Hart, W.G. Hymenopterous egg-pupal and larval-pupal parasitoids of Ceratitis capitata and Anastrepha spp. (Dip.: Tephritidae) in Costa Rica. Entomophaga 1981, 26, 285-290. [CrossRef]

31. Hernández-Ortiz, V.; Pérez-Alonso, R.; Wharton, R.A. Native parasitoids associated with the genus Anastrepha (Diptera: Tephritidae) in Los Tuxtlas, Veracruz, Mexico. Entomophaga 1994, 39, 171-178. [CrossRef]

32. Sivinski, J.; Aluja, M.; López, M. Spatial and temporal distributions of parasitoids of Mexican Anastrepha species (Diptera: Tephritidae) within the canopies of fruit trees. Ann. Entomol. Soc. Am. 1997, 90, 604-618. [CrossRef]

33. López, M.; Aluja, M.; Sivinski, J. Hymenopterous larval-pupal and pupal parasitoids of Anastrepha flies (Diptera: Tephritidae) in Mexico. Biol. Cont. 1999, 15, 119-129. [CrossRef]

34. Ovruski, S.; Aluja, M.; Sivinski, J.; Wharton, R. Hymenopteran parasitoids on fruit-infesting Tephritidae (Diptera) in Latin America and the southern United States: Diversity, distribution, taxonomic status and their use in fruit fly biological control. Integr. Pest Mgmt. Rev. 2000, 5, 81-107. [CrossRef]

35. Montoya, P.; Ayala, A.; López, P.; Cancino, J.; Cabrera, H.; Cruz, J.; Martinez, A.M.; Figueroa, I.; Liedo, P. Natural parasitism in fruit fly (Diptera: Tephritidae) populations in disturbed areas adjacent to commercial mango orchards in Chiapas and Veracruz, Mexico. Environ. Entomol. 2016, 45, 328-337. [CrossRef]

36. Hernández-Ortiz, V.; Manrique-Saide, P.; Delfín- González, H.; Novelo-Rincón, I. First report of Anastrepha compressa in Mexico and new records for other Anastrepha species in the Yucatan Peninsula (Diptera: Tephritidae). Fla. Entomol. 2002, 85, 389-391. [CrossRef]

37. Aluja, M.; Piñero, J.; López, M.; Ruíz, C.; Zúñiga, A.; Piedra, E.; Díaz-Fleisher, F.; Sivinski, J. New host plant and distribution records in Mexico for Anastrepha spp., Toxotrypana curvicauda Gerstacker, Rhagoletis zoqui Bush, Rhagoletis sp., and Hexachaeta sp. (Diptera: Tephritidae). Proc. Entomol. Soc. Wash. 2000, 102, 802-815.

38. Rzedowski, J. Vegetacion de Mexico; Limusa: Distrito Federal, Mexico, 1978; p. 432.

39. Miranda, F.D.P.; Hernández, X.E. Los tipos de vegetación de México y su clasificación. Bol. Soc. Bot. México 1963, 28, 29-179. [CrossRef]

40. Wharton, R.A.; Marsh, P.M.; Sharkey, M.J. Manual of the New World genera of the family Braconidae (Hymenoptera). Spec. Publ. Int. Soc. Hymenopterists 1997, 1, 459.

41. Tropicos.org. Missouri Botanical Garden. Available online: http://www.tropicos.org (accessed on 6 December 2018).

42. Garcí Ramírez, M.D.J.; Antonio Hernández, E.; Vargas Magaña, J.J.; Valencia Gutiérrez, M.D.C.; Chi Ruiz, J.C.; Placensia Valerio, Y. First host plant record for Anastrepha ampliata Hernández-Ortiz, 1990 (Diptera: Tephritidae). Biocyt Biol. Cienc. Tecnol. 2018, 11, 789-791.

43. Hernaández-Ortiz, V. El Género Anastrepha Schiner en México (Diptera: Tephritidae). Taxonomía, Distribución y sus Plantas Huéspedes; Publicacón No. 33; Instituto de Ecología: Xalapa, Mexico, 1992; p. 162.

44. Antonio Hernández, E.; Ramírez, M.D.J.G.; Lara, D.F. New records of the genus Anastrepha Schiner, 1868 (Diptera: Tephritidae) in the Isthmus of Tehuantepec, Oaxaca, Mexico. Biocyt Biol. Cienc. Tecnol. 2018, 11, 824-833. 
45. Sosa-Armenta, J.M.; López-Martínez, V.; Villegas-Torres, Ó.G.; Juárez-López, P.; Burgos-Solorio, A. Dinámica poblacional de moscas de la fruta en Quintana Roo, México. Southwest Entomol. 2017, 42, 275-282. [CrossRef]

46. Sosa-Armenta, J.M.; López-Martínez, V.; Alia-Tejacal, I.; García-Jiménez, D.; Guillen-Sánchez, D.; Delfín-González, H. Hosts of five Anastrepha species (Diptera: Tephritidae) in the state of Quintana Roo, Mexico. Fla. Entomol. 2015, 98, 1000-1002. [CrossRef]

47. Araujo, M.R.; Uramoto, K.; Ferreira, E.N.L.; Mesquita Filho, W.; Walder, J.M.M.; Savaris, M.; Zucchi, R.A. Fruit Fly (Diptera: Tephritidae) diversity and host relationships in diverse environments estimated with two sampling methods. Environ. Entomol. 2018, 48, 227-233. [CrossRef] [PubMed]

48. Plummer, C.C.; McPhail, M. The yellow chapote, a native host of the Mexican fruit fly. USDA Tech. Bull. 1941, 775, 1-12.

49. Bush, G.L. The cytotaxonomy of the larvae of some Mexican fruit flies in the genus Anastrepha (Tephritidae, Diptera). Psyche 1962, 69, 87-101. [CrossRef]

50. Villaseñor, J.L. Checklist of the native vascular plants of Mexico. Rev. Mex. Biodivers 2016, 87, 559-902. [CrossRef]

51. Thomas, D.B. Mexican fruit fly (Diptera: Tephritidae) and the phenology of its native host plant yellow chapote (Rutaceae) in Mexico. J. Entomol. Sci. 1999, 47, 1-16. [CrossRef]

52. Salter, E.A. Flora Nicaragüense. In Arboles y Arbustos Más Notables y el Uso de Sus Maderas y Otros Productos; Imprenta La Salle: Bluefield, Nicaragua, 1947; p. 280.

53. Pennington, T.D. Flora neotropica. Monograph 52. Sapotaceae; New York Botanical Garden for the Organization for Flora Neotropica: New York, NY, USA, 1990; p. 770.

54. Barbeau, G. Frutas Tropicales en Nicaragua. Edit. Ciencias Sociales. Managua, Nicaragua. 1990, p. 397. Available online: https://agris.fao.org/agris-search/search.do?recordID=NI2006000072 (accessed on 1 May 2020).

55. Bernays, E.A.; Chapman, R.F. Behavior: The Process of Host-Plant Selection. In Host-Plant Selection by Phytophagous Insects; Bernays, E.A., Chapman, R.F., Eds.; Chapman \& Hall: New York, NY, USA, 1994; pp. 95-165.

56. Brown, J.H.; Stevens, G.C.; Kaufman, D.M. The geographic range: Size, shape, boundaries, and internal structure. Annu. Rev. Ecol. Evol. Syst. 1996, 27, 597-623. [CrossRef]

57. Frankham, R.; Ballou, J.D.; Dudash, M.R.; Eldridge, M.D.B.; Fenster, C.B.; Lacy, R.C.; Mendelson, J.R.; Porton, I.J.; Ralls, K.; Ryder, O.A. Implications of different species concepts for conserving biodiversity. Biol. Conserv. 2012, 153, 25-31. [CrossRef]

58. Medianero, E.; Korytkowski, C.A.; Campo, C.; De León, C. Hymenoptera parasitoids associated with Anastrepha (Diptera: Tephritidae) at Cerro Jefe and Altos de Pacora, Panama. Rev. Colomb. Entomol. 2006, 32, 136-139.

59. Leyva, J.L.; Browning, H.W.; Gilstrap, F.E. Effect of host fruit species, size, and color on parasitization of Anastrepha ludens (Diptera: Tephritidae) by Diachasmimorpha longicaudata (Hymenoptera: Braconidae). Environ. Entomol. 1991, 20, 1469-1474. [CrossRef]

60. Sivinski, J.; Aluja, M. The roles of parasitoid foraging for hosts, food and mates in the augmentative control of Tephritidae. Insects 2012, 3, 668-691. [CrossRef]

61. Sivinski, J.M.; Calkins, C.O.; Baranowski, R.; Harris, D.; Brambila, J.; Diaz, J.; Burns, R.E.; Holler, T.; Dodson, G. Suppression of a Caribbean fruit fly (Anastrepha suspensa (Loew) Diptera: Tephritidae) population through augmented releases of the parasitoid Diachasmimorpha longicaudata (Ashmead) (Hymenoptera: Braconidae). Biol. Cont. 1996, 6, 177-185. [CrossRef]

62. Montoya, P.; López, P.; Cruz, J.; Lopez, F.; Cadena, C.; Cancino, J.; Liedo, P. Effect of Diachasmimorpha longicaudata releases on the native parasitoid guild attacking Anastrepha spp. larvae in disturbed zones of Chiapas, Mexico. BioControl 2017, 62, 581-593. [CrossRef]

63. Harbi, A.; Beitia, F.; Ferrara, F.; Chermiti, B.; Sabater-Muñoz, B. Functional response of Diachasmimorpha longicaudata (Ashmead) over Ceratitis capitata (Wiedemann): Influence of temperature, fruit location and host density. Crop. Prot. 2018, 109, 115-122. [CrossRef]

(C) 2020 by the authors. Licensee MDPI, Basel, Switzerland. This article is an open access article distributed under the terms and conditions of the Creative Commons Attribution (CC BY) license (http://creativecommons.org/licenses/by/4.0/). 